\title{
PAX NATO: THE OPPORTUNITIES \\ OF ENLARGEMENT
}

Raymond A. Millen

August 2002

\section{LUBRARY U. OF I. URBAMA-CHAMPAIGN}

